\title{
Optical Wireless Communications With Heterodyne Detection Over Turbulence Channels With Pointing Errors
}

\author{
Harilaos G. Sandalidis, Theodoros A. Tsiftsis, Member, IEEE, and George K. Karagiannidis, Senior Member, IEEE
}

\begin{abstract}
We study the error performance of an heterodyne differential phase-shift keying (DPSK) optical wireless (OW) communication system operating under various intensity fluctuations conditions. Specifically, it is assumed that the propagating signal suffers from the combined effects of atmospheric turbulence-induced fading, misalignment fading (i.e., pointing errors) and path-loss. Novel closed-form expressions for the statistics of the random attenuation of the propagation channel are derived and the bit-error rate (BER) performance is investigated for all the above fading effects. Numerical results are provided to evaluate the error performance of $\mathrm{OW}$ systems with the presence of atmospheric turbulence and/or misalignment. Moreover, nonlinear optimization is also considered to find the optimum beamwidth that achieves the minimum BER for a given signal-to-noise ratio value.
\end{abstract}

Index Terms-Atmospheric turbulence channel, bit-error rate (BER), heterodyne detection, misalignment fading, optical wireless (OW) communications, pointing errors.

\section{INTRODUCTION}

$\mathbf{O}$ PTICAL wireless (OW) communications, also known as free-space optical communications, suffer by various impairments as atmospheric turbulence and the misalignment fading (pointing errors). Moreover, the laser power is also attenuated as the distance increases [1]-[4].

Turbulence-induced fading, also known as scintillation, causes irradiance fluctuations in the received signals as a result of variations in the refractive index. Furthermore, building sway phenomena cause vibrations of the transmitter beam and, therefore, misalignment (pointing error effects). To keep line-of-sight between the transmitter and receiver, the designers should increase the beamwidth and power. However, a wide beamwidth increases the required signal-to-noise (SNR) ratio leading to increased cost and complexity, whereas a narrow one may result in outage appearances [5]. Hence, proper optimization techniques should be followed. Typical such studies include [5] and [6].

The combined effect of atmospheric turbulence and pointing error effects has been investigated in [7]-[10]. The majority

Manuscript received January 01, 2009; revised May 08, 2009. First published June 02, 2009; current version published August 28, 2009.

H. G. Sandalidis is with the Department of Computer Science and Biomedical Informatics, University of Central Greece, Papasiopoulou 2-4, GR-35100 Lamia, Greece (e-mail: sandalidis@ucg.gr).

T. A. Tsiftsis and G. K. Karagiannidis are with the Department of Electrical and Computer Engineering, Aristotle University of Thessaloniki, GR-54124 Thessaloniki, Greece (e-mail: thtsif@auth.gr; geokarag@auth.gr).

Digital Object Identifier 10.1109/JLT.2009.2024169 of these studies refer, however, to indirect modulation/direct detection (IM/DD) OW systems. Although direct detection is the main mode of detection in OW systems, coherent communications have been also proposed as an alternative detection mode. Heterodyne detection is a more complicated detection method but has the ability to overcome the thermal noise effect. The performance of such systems over atmospheric turbulence have also been evaluated in the past. In [11], Kiasaleh has studied the bit-error-rate (BER) performance of an OW heterodyne differential phase-shift keying (DPSK) communication system in the presence of $\mathrm{K}$ turbulence fading whereas in [12], Tsiftsis has presented a closed-form expression of the BER over gamma-gamma turbulence channels.

In this paper, we consider an OW heterodyne DPSK system over channels that are characterized by either atmospheric turbulence, or by pointing error effects, or by the combined effect of atmospheric path-loss with the presence of the above two factors. Scintillation is modeled using the gamma-gamma distribution and atmospheric loss is determined by the exponential Beers-Lambert Law. Furthermore, we provide closed-form expressions for the statistics of the above combined attenuation factors and we investigate the BER performance. In order to find the optimum beamwidth that gives the minimum BER for given SNR values, we use nonlinear optimization taking into account various metrics such as the beamwidth, the average SNR and the normalized jitter.

The remainder of the paper is organized as follows: Section II briefly describes the system model under consideration. The channel state which models the combined effects of path-loss, turbulence and misalignment fading, is also described. New probability density function (pdf) and cumulative distribution function (cdf) incorporating the combined effect are obtained in closed form. Section III presents closed-form expressions for the average BER of the DPSK system over gamma-gamma turbulence, or misalignment fading, or in the presence of the combined attenuation factors. In Section IV, proper graphs to verify the accuracy of the derived expressions and the assumptions followed during the optimization method, are presented. Finally, some concluding remarks are given in Section V.

\section{System AND CHANNEl MODEL}

We consider an OW system with DPSK heterodyne detection. DPSK has recently received considerable attention in OW research since it attains a $3 \mathrm{~dB}$ sensitivity benefit over on-off keying $(\mathrm{OOK})$, and reduced peak power which mitigates any nonlinear effects [13]. For these reasons it has been proposed as 
an effective modulation scheme for terrestrial [14] as well as for satellite optical systems [15]. ${ }^{1}$

The laser beams propagate along a horizontal path through a gamma-gamma turbulence channel with additive white Gaussian noise (AWGN) in the presence of pointing errors. DPSK demodulators determine the changes in the phase of the received signals and, therefore, such schemes depend on the difference between successive phases [17]. For that reason we need a quasi-static channel assumption where the irradiance remains constant over a block of $N$ channel symbols and is statistically independent between the blocks. Without loss of generality, a constant channel condition for at least two consecutive bit intervals is assumed, where the channel statistics remains unchanged [11]. Therefore, the impact of phase noise is not considered in this study since we consider a negligible phase noise accumulation over two consecutive bit intervals.

The channel state is considered to be a product of three factors i.e., $h=h_{l} h_{a} h_{p}$, where $h_{l}$ is the deterministic path loss, $h_{a}$ is the random attenuation due to atmospheric turbulence, and $h_{p}$ is the random attenuation due to geometric spread and pointing errors [9]. We particularly assume that the pointing error is due to building sway, with sub-Hz bandwidth (correlation times of a few seconds). Note that scintillation has a much smaller correlation time (10-100 ms) as compared with building sways, and, therefore, the two above impairments are considered to be independent. Pointing errors due to vibrations, induced primarily by wind, as well as beam tilt and wander, though critical, are not considered in this work. The mathematical expressions of the above impairments are presented below in detail.

\section{A. Atmospheric Loss}

The atmospheric loss is determined by the exponential BeersLambert Law as

$$
h_{l}(z)=\exp (-\sigma z)
$$

where $h_{l}(z)$ is the loss over a propagation path of length $z$ and $\sigma$ is the attenuation coefficient. The atmospheric loss depends on visibility which can be measured directly from the atmosphere. The attenuation is considered constant during a long time period [9].

\section{B. Atmospheric Turbulence}

The gamma-gamma turbulence model has the following pdf [1]

$f_{h_{a}}\left(h_{a}\right)=\frac{2(\alpha \beta)^{(\alpha+\beta) / 2}}{\Gamma(\alpha) \Gamma(\beta)} h_{a}^{(\alpha+\beta / 2)-1} K_{\alpha-\beta}\left(2 \sqrt{\alpha \beta h_{a}}\right) h_{a}>0$

where $h_{a}$ is the normalized irradiance, $\Gamma(\cdot)$ is the well-known Gamma function [18, eq. (8.310.1)], $K_{\nu}(\cdot)$ is the $\nu$ th-order modified Bessel function of the second kind [18, eq. (8.432.2)], and $\alpha, \beta$ are the effective number of small-scale and large-scale eddies of the scattering environment, respectively [19].

\footnotetext{
${ }^{1}$ For more information on feasibility of such a modulation scheme for OW usage, see also [16].
}

The parameters $\alpha$ and $\beta$ can be directly related to the atmospheric conditions. Assuming plane wave propagation, the parameters $\alpha$ and $\beta$ for a point receiver and zero inner scale are given by

$$
\begin{aligned}
& \alpha=\left[\exp \left(\frac{0.49 \sigma_{R}^{2}}{\left(1+1.11 \sigma_{R}^{12 / 5}\right)^{7 / 6}}\right)-1\right]^{-1} \\
& \beta=\left[\exp \left(\frac{0.51 \sigma_{R}^{2}}{\left(1+0.69 \sigma_{R}^{12 / 5}\right)^{5 / 6}}\right)-1\right]^{-1} .
\end{aligned}
$$

The unitless parameter $\sigma_{R}^{2}$ is called Rytov variance and is used as a metric of the strength of turbulence fluctuations [1]. It is also given by [9]

$$
\sigma_{R}^{2}=1.23 C_{n}^{2} k^{7 / 6} L^{11 / 6}
$$

where $C_{n}^{2}$ denotes the index of refraction structure parameter which is considered constant for horizontal paths, $k=2 \pi / \lambda$ is the optical wave number, $\lambda$ is the wavelength, and $L$ is the propagation distance [1].

\section{Misalignment Fading}

The pdf of $h_{p}$ can be derived using the assumptions and methodology described in [9]. Specifically, if we assume a Gaussian spatial intensity profile of beam waist $w_{z}$ (radius calculated at $e^{-2}$ ) on the receiver plane at distance $z$ from the transmitter and a circular aperture of radius $r$, the fraction of the collected power due to geometric spread with radial displacement $\alpha$ from the origin of the detector can be approximated as [9]

$$
h_{p}(\alpha) \approx A_{0} \exp \left(-\frac{2 \alpha^{2}}{w_{z_{e q}}^{2}}\right)
$$

where $w_{z_{e q}}^{2}=w_{z}^{2} \sqrt{\pi} \operatorname{erf}(v) / 2 v \exp \left(-v^{2}\right), v=\sqrt{\pi} r / \sqrt{2} w_{z}$, $A_{0}=[\operatorname{erf}(v)]^{2}$ with $\operatorname{erf}(\cdot)$ being the error function $[20$, eq. (7.1.1)]. By considering independent identical Gaussian distributions for the elevation and the horizontal displacement (sway), the radial displacement $\alpha$ follows a Rayleigh distribution. Then, the pdf of $h_{p}$ is given by

$$
f_{h_{p}}\left(h_{p}\right)=\frac{\gamma^{2}}{A_{0}^{\gamma^{2}}} h_{p}^{\gamma^{2}-1}, \quad 0 \leq h_{p} \leq A_{0}
$$

where $\gamma=w_{z_{e q}} / 2 \sigma_{s}$ is the ratio between the equivalent beam radius at the receiver and the pointing error displacement standard deviation (jitter) at the receiver (see [9] for more details).

\section{Statistics of the Combined Attenuation}

Using the previous pdfs for turbulence and misalignment fading, the pdf of $h=h_{l} h_{a} h_{p}$ is given as [9]

$$
f_{h}(h)=\int f_{h \mid h_{a}}\left(h \mid h_{a}\right) f_{h_{a}}\left(h_{a}\right) d h_{a}
$$


where $f_{h \mid h_{a}}\left(h \mid h_{a}\right)$ is the conditional probability given $h_{a}$ state and is expressed by

$$
\begin{aligned}
f_{h \mid h_{a}}\left(h \mid h_{a}\right) & =\frac{1}{h_{a} h_{l}} f_{h_{p}}\left(\frac{h}{h_{a} h_{l}}\right) \\
& =\frac{\gamma^{2}}{A_{0} \gamma^{2} h_{a} h_{l}}\left(\frac{h}{h_{a} h_{l}}\right)^{\gamma^{2}-1} \quad 0 \leq h \leq A_{0} h_{a} h_{l} .
\end{aligned}
$$

By substituting (2) and (8) in (7), $f_{h}(h)$ is given by

$$
\begin{aligned}
& f_{h}(h)=\frac{2 \gamma^{2}(\alpha \beta)^{(\alpha+\beta / 2)}}{\left(A_{0} h_{l}\right)^{\gamma^{2}} \Gamma(\alpha) \Gamma(\beta)} h^{\gamma^{2}-1} \\
& \quad \times \int_{h / A_{0} h_{l}}^{\infty} h_{a}^{(a+\beta / 2)-1-\gamma^{2}} K_{\alpha-\beta}\left(2 \sqrt{\alpha \beta h_{a}}\right) d h_{a} .
\end{aligned}
$$

The above integral was computed numerically in [9, eq. (15)]. In order to provide a closed-form solution, we express the $K_{\nu}(\cdot)$ in terms of the Meijer's G-function $G_{p, q}^{m, n}[\cdot]$, defined in [18, eq. (9.301)]. By using [21, eq. (14)] and [22, eq. (02.24.2.3)], the $K_{\nu}(\cdot)$ can be written as

$$
K_{\nu}(x)=\frac{1}{2} G_{0,2}^{2,0}\left[\left.\frac{x^{2}}{4}\right|_{(\nu / 2),-(\nu / 2)} ^{-}\right] .
$$

Then

$$
\begin{aligned}
& f_{h}(h)=\frac{\alpha \beta \gamma^{2}}{A_{0} h_{l} \Gamma(\alpha) \Gamma(\beta)}\left(\frac{\alpha \beta h}{A_{0} h_{l}}\right)^{(\alpha+\beta / 2)-1} \\
& \times G_{1,3}^{3,0}\left[\begin{array}{cc}
\frac{\alpha \beta}{A_{0} h_{l}} h & 1-\frac{\alpha+\beta}{2}+\gamma^{2} \\
-\frac{\alpha+\beta}{2}+\gamma^{2}, \frac{\alpha-\beta}{2}, \frac{\beta-\alpha}{2}
\end{array}\right] .
\end{aligned}
$$

Equation (11) can be further simplified using [18, eq. (9.31.5)] as

$$
\begin{aligned}
& f_{h}(h)=\frac{\alpha \beta \gamma^{2}}{A_{0} h_{l} \Gamma(\alpha) \Gamma(\beta)} \\
& \times G_{1,3}^{3,0}\left[\frac{\alpha \beta}{A_{0} h_{l}} h \mid \begin{array}{c}
\gamma^{2} \\
\gamma^{2}-1, \alpha-1, \beta-1
\end{array}\right] .
\end{aligned}
$$

Note that Meijer's G-function is a standard built-in function in most of the well-known mathematical software packages such as Maple, Mathematica, etc. In addition, using [21, eq. (18)], Meijer's G-function can be written in terms of the more familiar generalized hypergeometric functions [18, eq. (9.14.1)]. Thus,
(12) is equivalently expressed by (13), shown at the bottom of the page, where ${ }_{1} F_{2}(\cdot ; \cdot, \cdot ;)$ is the generalized hypergeometric function defined in [18, eq. (9.14)].

See equation (13), shown at the bottom of the page.

The cdf of $h$ results from (12) as

$$
F_{h}(h) \triangleq \int_{0}^{h} f_{h}(h) d h .
$$

The integral can be solved by expressing the $K_{\nu}(\cdot)$ in terms of the Meijer's G-function and using [21, eq. (26)] and [18, eq. (9.31.5)] leading to

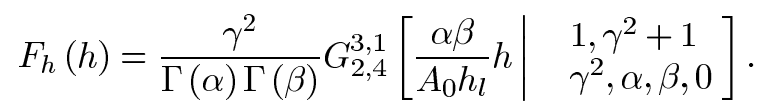

Similarly, the above equation can also be expressed in terms of generalized hypergeometric functions.

\section{Average BER}

The conditional on signal intensity BER of a shot-noise limited heterodyne DPSK system is given by [11]

$$
P(e \mid h)=\frac{1}{2} e^{-\zeta(h)}
$$

where $\zeta(h)$ is the signal intensity-dependent SNR given by

$$
\zeta(h)=\frac{\eta_{e} A T_{s} h}{h_{P} v} .
$$

In the above equation $\eta_{e}$ is the quantum efficiency of the detector, $A$ is the detector area in square meters, $T_{s}$ is the DPSK symbol duration in seconds, $h_{P}$ is the Planck's constant in Joules/Hertz, $v$ is the frequency of the optical signal in Hertz. Note that we follow the same assumptions as in [11] and particularly consider that time variations in irradiance over a symbol time is assumed away. That is, we assume that duration of $T_{s}$ is smaller than the coherence time of the channel, having thus a constant amplitude scenario for the received signal. For a slow-fading environment, the average BER, $P(e)$, can be obtained by averaging (16) over the pdf of $h$, i.e.,

$$
P(e)=\int_{0}^{\infty} P(e \mid h) f_{h}(h) d h .
$$

$$
\begin{aligned}
f_{h}(h)= & \frac{\pi^{2} \gamma^{2} \csc \left(\pi\left(a-\gamma^{2}\right)\right) \csc \left(\pi\left(\beta-\gamma^{2}\right)\right)\left(\frac{\alpha \beta h}{A_{0} h_{l}}\right)^{\gamma^{2}}}{h \Gamma(a) \Gamma(\beta) \Gamma\left(\gamma^{2}-a+1\right) \Gamma\left(\gamma^{2}-\beta+1\right)} \\
& +\frac{\pi^{2} \gamma^{2} \csc ((\beta-a) \pi) \csc \left(\pi\left(\gamma^{2}-a\right)\right)_{1} F_{2}\left(a-\gamma^{2} ; a-\beta+1,-\gamma^{2}+a+1 ; \frac{a \beta h}{A_{0} h_{l}}\right)\left(\frac{a \beta h}{A_{0} h_{l}}\right)^{a}}{h \Gamma(a) \Gamma(a-\beta+1) \Gamma(\beta) \Gamma\left(-\gamma^{2}+a+1\right) \Gamma\left(\gamma^{2}-a+1\right)} \\
& +\frac{\pi^{2} \gamma^{2} \csc ((a-\beta) \pi) \csc \left(\pi\left(\gamma^{2}-\beta\right)\right)_{1} F_{2}\left(\beta-\gamma^{2} ;-a+\beta+1,-\gamma^{2}+\beta+1 ; \frac{a \beta h}{A_{0} h_{l}}\right)\left(\frac{a \beta h}{A_{0} h_{l}}\right)^{\beta}}{h \Gamma(a) \Gamma(\beta) \Gamma(-a+\beta+1) \Gamma\left(-\gamma^{2}+\beta+1\right) \Gamma\left(\gamma^{2}-\beta+1\right)}
\end{aligned}
$$


Next, we evaluate the BER considering atmospheric turbulence, or misalignment fading, or the combined effect of the previous signal attenuation factors, respectively. For each case, the BER is derived in terms of the average SNR which is defined as

$$
\mu=E[\zeta(h)]=\frac{\eta_{e} A T_{s}}{h_{P} v} \cdot E[h]
$$

where $E[\cdot]$ denotes expectation.

\section{A. Atmospheric Turbulence}

In case only atmospheric turbulence exists, $h=h_{a}$. Since $h_{a}$ is normalized, $E[h]=1$ and, hence, $\mu=\left(\eta_{e} A T_{s} / h_{P} v\right)$. By substituting (2) in (18), the average BER can be easily extracted as in [12], given by

$$
P(e)=\frac{1}{2 \Gamma(\alpha) \Gamma(\beta)} G_{1,2}^{2,1}\left[\frac{\alpha \beta}{\mu} \mid \begin{array}{l}
1 \\
\alpha, \beta
\end{array}\right] .
$$

\section{B. Misalignment Fading}

In case where only misalignment fading exists, $h=h_{p}$. It is easy to show that $E\left[h_{p}\right]=\left(A_{0} \gamma^{2} / \gamma^{2}+1\right)$ and, hence, $\mu=\left(\eta_{e} A T_{s} / h_{P} v\right)\left(A_{0} \gamma^{2} / \gamma^{2}+1\right)$. The BER is then derived by substituting (6) in (18). That is

$$
P(e)=\frac{1}{2} \int_{0}^{A_{0}} \frac{\gamma^{2}}{A_{0}^{\gamma^{2}}} h^{\gamma^{2}-1} e^{-\mu h\left(\gamma^{2}+1 / A_{0} \gamma^{2}\right)} d h .
$$

A closed-form solution for BER is obtained using [18, eq. (3.381.1)] as

$$
P(e)=\frac{\gamma^{2}\left(\mu \frac{\gamma^{2}+1}{\gamma^{2}}\right)^{-\gamma^{2}}}{2}\left[\Gamma\left(\gamma^{2}\right)-\Gamma\left(\gamma^{2}, \mu \frac{\gamma^{2}+1}{\gamma^{2}}\right)\right]_{(22)}
$$

where $\Gamma(\cdot, \cdot)$ is the upper incomplete Gamma function defined in $[18$, eq. (8.350.2)].

\section{Combined Attenuation}

In that case, it is easily found using (12) that $E[h]=$ $h_{l}\left(A_{0} \gamma^{2} / \gamma^{2}+1\right)$. Therefore, the BER is derived by substituting (12) in (18), i.e.,

$$
\begin{aligned}
P(e)= & \int_{0}^{\infty} \frac{\alpha \beta \gamma^{2}}{2 A_{0} h_{l} \Gamma(\alpha) \Gamma(\beta)} \\
& \times G_{1,3}^{3,0}\left[\frac{\alpha \beta}{A_{0} h_{l}} h \mid \gamma^{2}-1, \alpha-1, \beta-1\right] \\
& \times G_{0,1}^{1,0}\left[\left.\mu h \frac{\gamma^{2}+1}{h_{l} A_{0} \gamma^{2}}\right|_{0} ^{-}\right] d h .
\end{aligned}
$$

A closed-form solution for BER is obtained using [18, eq. (7.813.1)] and [18, eq. (9.31.5)] as

$$
P(e)=\frac{\gamma^{2}}{2 \Gamma(\alpha) \Gamma(\beta)} G_{2,3}^{3,1}\left[\begin{array}{l|l}
\alpha \beta \gamma^{2} & \begin{array}{l}
1, \gamma^{2}+1 \\
\mu\left(\gamma^{2}+1\right)
\end{array} \\
\gamma^{2}, \alpha, \beta
\end{array}\right] .
$$

\section{NUMERICAL RESULTS AND DISCUSSION}

In Fig. 1 the average BER is depicted in terms of the average SNR for various values of turbulence strength, $\sigma_{R}^{2}$, where only the atmospheric turbulence effect is assumed. We particularly

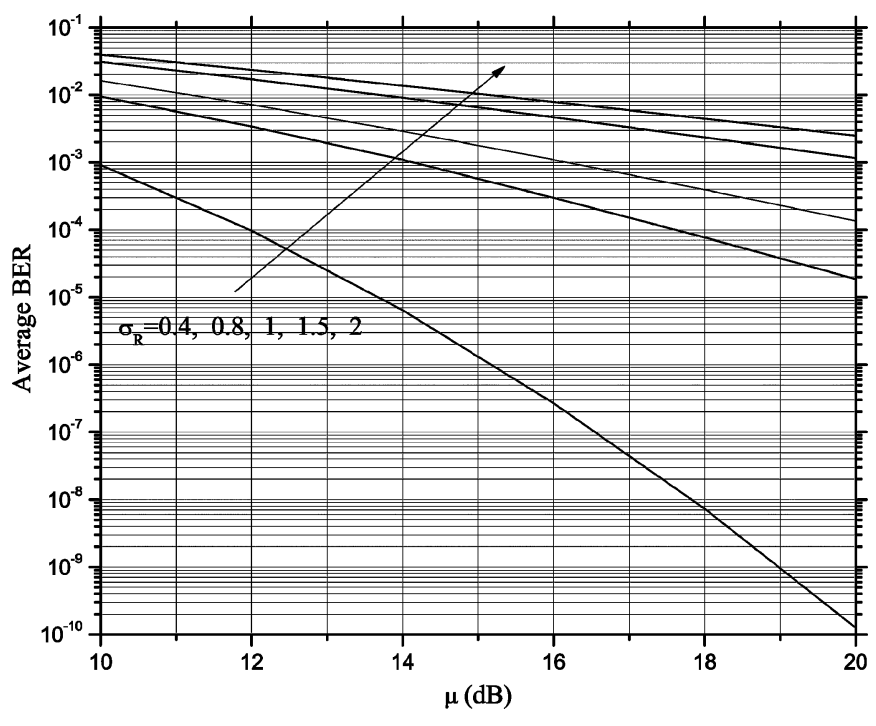

Fig. 1. BER versus average SNR assuming turbulence effect only.

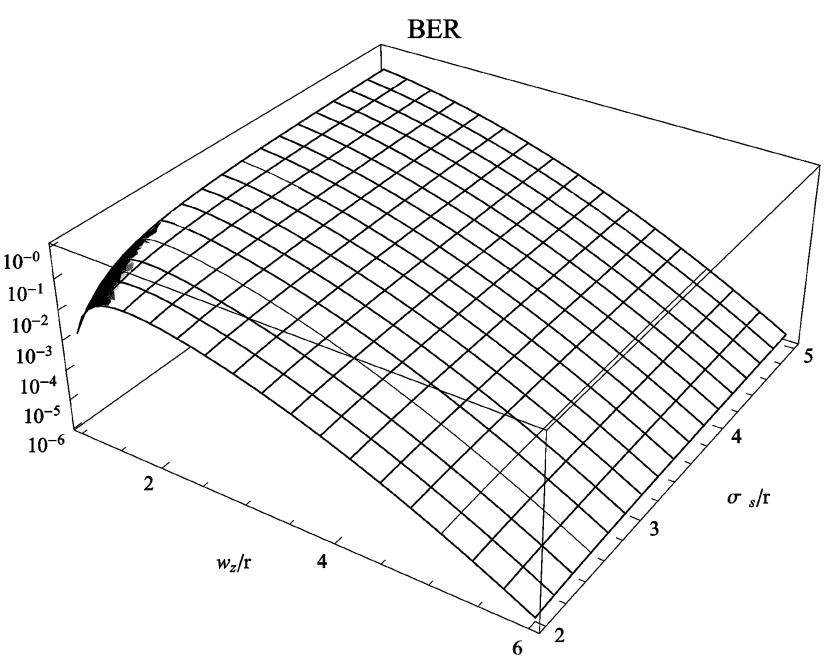

Fig. 2. BER versus normalized beamwidth and normalized jitter assuming pointing error effects only.

use the following values that represent weak and strong turbulence conditions: $\sigma_{R 1}=0.4, \sigma_{R 2}=0.8, \sigma_{R 3}=1, \sigma_{R 3}=1.5$, and $\sigma_{R 4}=2$. As it was expected, the BER increases as turbulence strength gets stronger. Note that the curves in this figure are valid for any combination of distance length, wavelength and structure parameter $C_{n}^{2}$.

Next, we only assume pointing error effects and a constant average SNR, $\mu=30 \mathrm{~dB}$. The normalized beamwidth, $w_{z} / r$, is assumed to vary between 1 and 6 whereas the normalized jitter, $\sigma_{s} / r$, between 2 and 5. It is shown in Fig. 2 that the BER performance depends on these two factors. Similar BER plots can be depicted for the combined attenuation model. For example, Fig. 3 shows a 3-D plot of BER against turbulence strength and normalized beamwidth assuming $\mu=50 \mathrm{~dB}$ whereas in Fig. 4, the BER in terms of the average SNR and the normalized beamwidth for turbulence strength $\sigma_{R}^{2}=4$, is plotted. In previous two figures, we assumed a normalized jitter value, $\sigma_{s} / r=3$, and a constant path loss factor, $h_{l}=0.9$, which is a typical value for clear weather conditions [23]. 


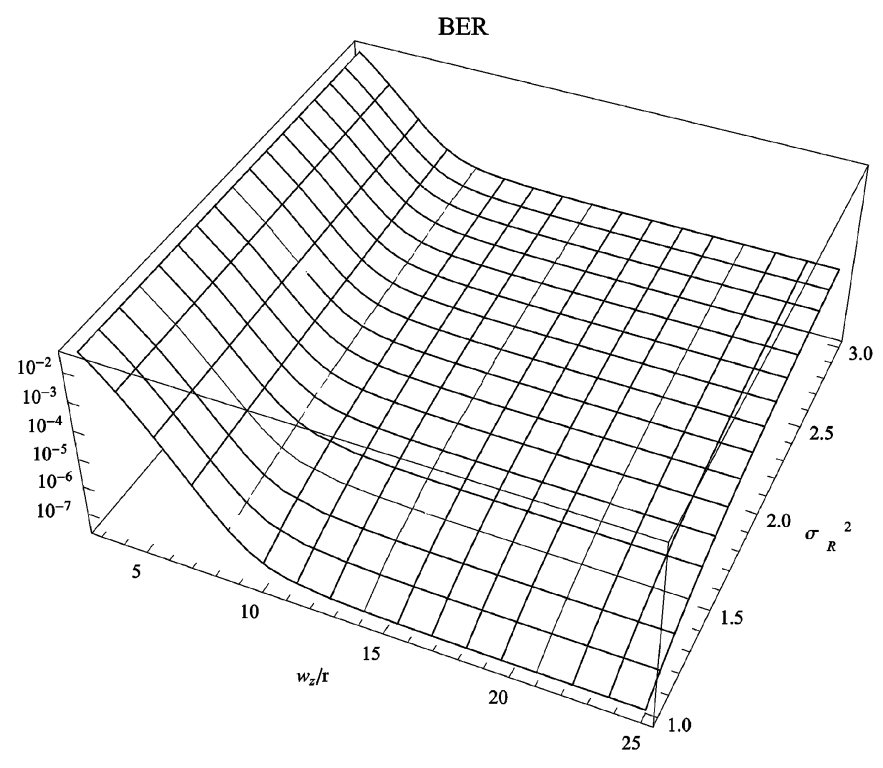

Fig. 3. BER versus normalized beamwidth and turbulence strength.

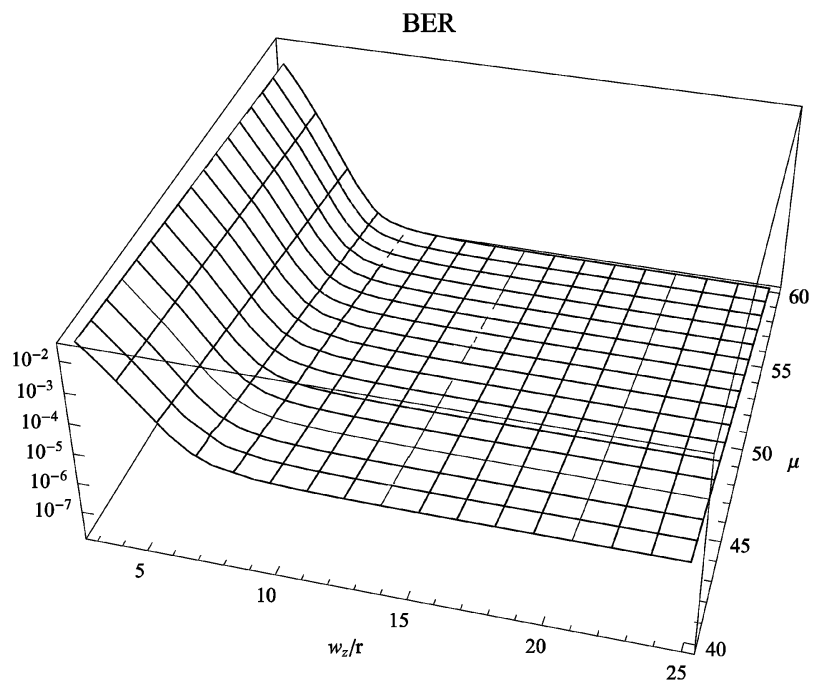

Fig. 4. BER versus normalized beamwidth and average SNR.

Considering all the above fading models which affect the average BER, a target BER can, therefore, be achieved by selecting an optimum beamwidth which minimizes the required SNR. ${ }^{2}$ As the problem is nonlinear and contains complex mathematical expressions we solve it numerically using a direct search method such as the Nelder-Mead one. The method uses the concept of a simplex, which is a polytope of $N+1$ vertices in $N$ dimensions. At first, the function is evaluated at the vertices of a simplex. Then, as better points are found, the simplex is iteratively shrunk until a desired bound is obtained [24]. The optimum beamwidth is chosen between the extreme points 1 and 25 where the beamwidth is set to vary.

In Fig. 5, the minimum BER is calculated for optimum normalized beamwidth values. In this graph, one can easily find the combination of minimum BER and optimized beamwidth for a

\footnotetext{
${ }^{2}$ The problem can be stated as follows: for a given average SNR, find the optimum beamwidth that achieves the minimum BER.
}

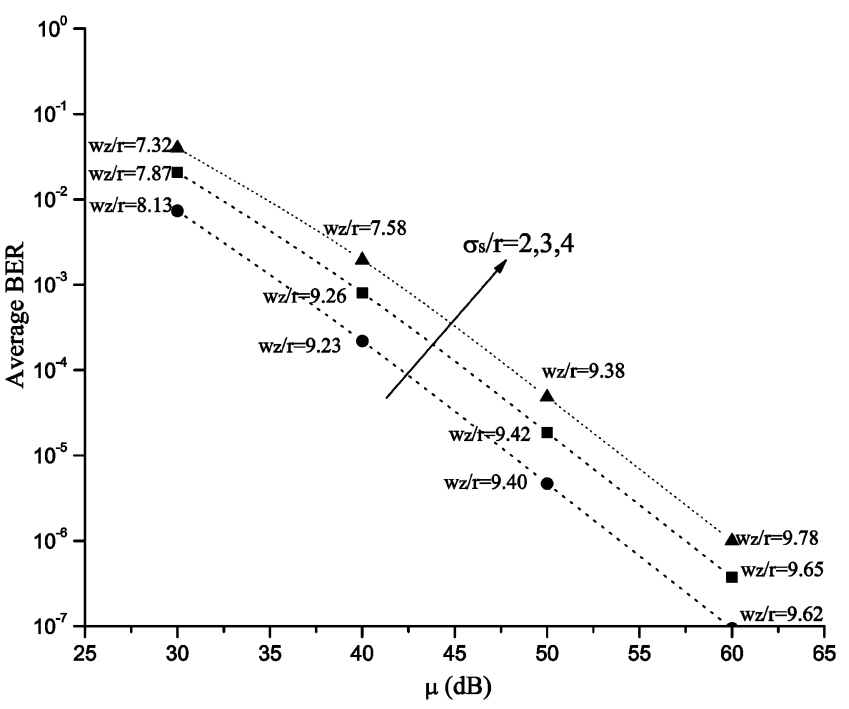

Fig. 5. Optimum normalized beamwidth values for achieving minimum BER.

given SNR value. For example, a minimum BER of 0.01 occurs for $w_{z} / r=8.13, \sigma_{s} / r=2$, and $\mu=30 \mathrm{~dB}$.

\section{CONClusions AND DiReCTIONS FOR FURTHER RESEARCH}

Coherent communications have been proposed as an alternative detection mode for OW systems. In this paper, we examined the BER performance of an OW communication system with DPSK heterodyne detection. We derived BER expressions over atmospheric turbulence and pointing error effects separately. A novel fading distribution including the combined effects of path-loss, turbulence, and pointing errors was analytically derived and a closed-form expression of the error performance, was presented. Optimized beamwidth values to achieve the minimum BER for various SNR values, were also presented.

It is also of great interest to further evaluate the performance of the proposed system using more complicated models. Such models may involve phase noise as well as beam wander and tilt consideration effects. Variations in phase occurs when the characteristics of the channel do not remain constant for at least two consecutive bit intervals and lead to phase noise appearance. Moreover, beam wander and tilt effects have bandwidths of a few $\mathrm{Hz}$, which is comparable in correlation time to the scintillation characteristics of the channel, and, therefore, there is an inherent dependency between pointing jitter and scintillation in OW environment due to the dependency of such process on random wind velocity [25]. Finally, by considering all the above as well as the use of error control coding and/or transmit receive diversity in order to increase BER performance, this paper may contribute to a number of open issues for future investigation.

\section{REFERENCES}

[1] L. Andrews, R. L. Philips, and C. Y. Hopen, Laser Beam Scintillation with Applications. Bellingham, WA: SPIE, 2001.

[2] J. T. Li and M. Uysal, "Optical wireless communications: System model, capacity and coding," in Proc. IEEE Vehicular Technological Conf., Orlando, FL, Oct. 2003, pp. 168-172.

[3] M. Uysal, S. M. Navidpour, and J. T. Li, "Error rate performance of coded free-space optical links over strong turbulence channels," IEEE Commun. Lett., vol. 8, pp. 635-637, Oct. 2004. 
[4] E. Bayaki, R. Schober, and R. K. Mallik, "Performance analysis of free-space optical systems in gamma-gamma fading," presented at the IEEE Global Commun. Conf. (GLOBECOM), New Orleans, LA, Dec. 2008.

[5] S. Arnon, "Optimization of urban optical wireless communications systems," IEEE Trans. Wireless Commun., vol. 2, no. 7, pp. 626-629, Jul. 2003.

[6] H. G. Sandalidis, "Optimization models for misalignment fading mitigation in optical wireless links," IEEE Commun. Lett., vol. 12, pp. 395-397, 2008.

[7] K. Kiasaleh, "On the probability density function of signal intensity in free-space optical communication systems impaired by pointing jitter and turbulence," Opt. Eng., vol. 33, pp. 3748-3757, 1994.

[8] S. Arnon, "Effects of atmospheric turbulence and building sway on optical wireless-communication systems," Opt. Lett., vol. 28, pp. 129-131, 2003.

[9] A. A. Farid and S. Hranilovic, "Outage capacity optimization for freespace optical links with pointing errors," J. Lightw. Technol., vol. 25, pp. 1702-1710, Jul. 2007.

[10] H. G. Sandalidis, T. A. Tsiftsis, G. K. Karagiannidis, and M. Uysal, "BER performance of FSO links over strong atmospheric turbulence channels with pointing errors," IEEE Commun. Lett., vol. 1, no. 1, pp. 44-46, Jan. 2008.

[11] K. Kiasaleh, "Performance of coherent DPSK free-space optical communication systems in K-distributed turbulence," IEEE Trans. Commun., vol. 54, no. 4, pp. 604-607, Apr. 2006.

[12] T. A. Tsiftsis, "Performance of heterodyne wireless optical communication systems over gamma-gamma atmospheric turbulence channels," Electron. Lett., vol. 44, Feb. 2008.

[13] D. O. Caplan, "Laser communication transmitter and receiver design," J. Opt. Fiber Commun. Rep., vol. 4, pp. 225-362, 2007.

[14] W. O. Popoola, Z. Ghassemlooy, and E. Leitgeb, "BER performance of DPSK subcarrier modulated free space optics in fully developed speckle," in Proc. 6th Int. Symp. Communication Systems, Networks and Digital Signal Processing, 2008, pp. 273-277.

[15] M. Antonini, S. Betti, V. Carrozzo, E. Duca, and M. Ruggieri, "Feasibility analysis of a HAP-LEO optical link for data relay purposes," presented at the IEEE Aerospace Conf., 2006.

[16] D. O. Caplan, "Multi-Channel DPSK Receiver," U.S. patent 7233430, 19-06-2007.

[17] A. B. Carlson, P. B. Crilly, and J. C. Rutledge, Communication Systems, 4th ed. New York: McGraw-Hill, 2002.

[18] I. S. Gradshteyn and I. M. Ryzhik, Table of Integrals, Series, and Products, 7th ed. New York: Academic, 2008.

[19] M. Uysal, J. T. Li, and M. Yu, "Error rate performance analysis of coded free-space optical links over gamma-gamma turbulence channels," IEEE Trans. Wireless Commun., vol. 5, no. 6, pp. 1229-1233, Jun. 2006.

[20] M. Abramovitz and I. A. Stegun, Handbook of Mathematical Functions With Formulas, Graphs, and Mathematical Tables, 9th ed. New York: Dover, 1972.

[21] V. S. Adamchik and O. I. Marichev, "The algorithm for calculating integrals of hypergeometric type functions and its realization in REDUCE system," in Proc. Int. Conf. Symbolic and Algebraic Computation, Tokyo, Japan, 1990, pp. 212-224.

[22] A. P. Prudnikov, Y. A. Brychkov, and O. I. Marichev, Integrals and Series. More Special Functions. London, U.K.: Gordon and Breach, 1992, vol. 3 .

[23] I. Kim, B. McArthur, and E. Korevaar, "Comparison of laser beam propagation at $785 \mathrm{~nm}$ and $1550 \mathrm{~nm}$ in fog and haze for optical wireless communications," in Proc. SPIE, Feb. 2001, vol. 4214, pp. 26-37.

[24] J. A. Nelder and R. Mead, "A simplex method for function minimization," The Comput. J., vol. 7, pp. 308-313, 1965.

[25] L. C. Andrews and R. L. Philips, "Recent results on optical scintillation in the presence of beam wander," in Proc. SPIE, 2008, vol. 6878

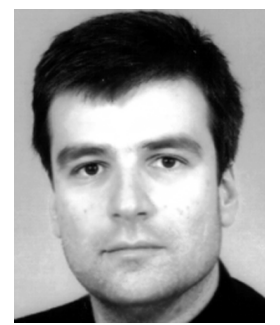

Harilaos G. Sandalidis was born in Florina, Greece, in 1972. He received the five year Diploma degree in electronics and computer engineering and the M.Sc. degree in business administration from the Production Engineering and Management Department, Technical University of Crete, Greece, in 1995 and 1998, respectively. He also received the M.Sc. degree in radiofrequency and microwave communications and the Ph.D. degree in the telecommunications area from the Electronics and Telecommunications (former Electronics and Electrical engineering) Department, University of Bradford, U.K., in 1996 and 2002, respectively.

During the period between 1996 and 2001, he was a Research Assistant at the Telecommunications Systems Institute of Crete, Greece, working towards the Ph.D. degree in collaboration with Bradford University. After his military service, he joined TEMAGON (former OTE Consulting) in 2002 where he worked as a telecom consultant for the risk mitigation program for the 2004 Olympic Telecommunication Network in collaboration with Telcordia Technologies, Inc. $\mathrm{He}$ also worked as a Senior Investigator in the Greek Ombudsman. In March 2009, he joined University of Central Greece, where he is a Lecturer in the Department of Computer Science and Biomedical Informatics. His major research interests include optical wireless communications, computational intelligence, and heuristic optimization techniques regarding their application to the telecommunications field.

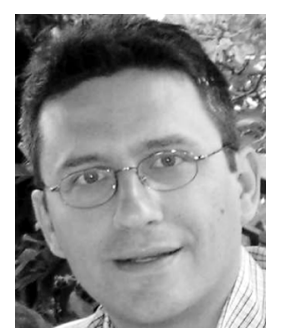

Theodoros A. Tsiftsis (S'02-M'04) was born in Lamia, Greece, in November 1970. He received the degree in physics from the Aristotle University of Thessaloniki, Greece, in 1993, the M.Sc. degree in electrical engineering from the Heriot-Watt University, Edinburgh, U.K., in 1995, the M.Sc. degree in decision sciences from the Athens University of Economics and Business, Greece, in 2000, and the Ph.D. degree in electrical engineering from the University of Patras, Greece, in 2005.

$\mathrm{He}$ is currently an Adjunct Assistant Professor in the Department of Electrical Engineering, Technological Educational Institute of Lamia, Greece, and he is also a visiting scientist in the Wireless Communications Systems Group (WCSG), Department of Electrical and Computer Engineering, Aristotle University of Thessaloniki, Greece. His major research interests include cooperative communications, wireless communications theory, performance analysis over fading channels, and free-space optical communications.

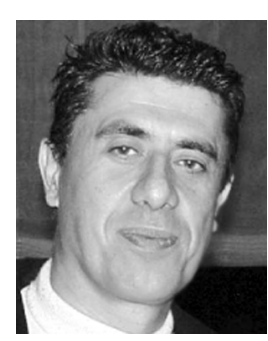

George K. Karagiannidis (M'97-SM'04) was born in Pithagorion, Samos Island, Greece. He received the University and Ph.D. degrees in electrical engineering from the University of Patras, Patras, Greece, in 1987 and 1999, respectively.

From 2000 to 2004, he was a Senior Researcher at the Institute for Space Applications and Remote Sensing, National Observatory of Athens, Greece. In June 2004, he the joined Aristotle University of Thessaloniki, Thessaloniki, Greece, where he is currently an Associate Professor of digital communications systems in the Electrical and Computer Engineering Department. His current research interests include wireless communication theory, digital communications over fading channels, cooperative communications, wireless optical communications, and underwater communications. He is the author or coauthor of more than 90 technical papers published in scientific journals and presented at international conferences. He is also a coauthor of two chapters in books and a coauthor of the Greek edition of a book on mobile communications.

Dr. Karagiannidis has been a member of Technical Program Committees for several IEEE conferences. He is a member of the editorial boards of the IEEE TRANSACTIONS ON COMMUNICATIONS and the IEEE COMMUNICATIONS LETTERS and Guest Editor for the Special Issue on Optical Wireless Communications of the IEEE JOURNAL ON SELECTED AREAS IN COMMUNICATIONS. He also serves on the editorial board of the EURASIP Journal on Wireless Communications and Networking. He is co-recipient of the Best Paper Award of the Wireless Communications Symposium (WCS) in IEEE International Conference on Communications (ICC' 07), Glasgow, U.K., June 2007. 